Results End of year 1 showed a 68\% improvement to Good out of those who had attended the training. Working together to share ideas to improve quality - 'a really good initiative' CQC Area Manager; 'It has inspired and raised my confidence to pursue an outstanding rating. It is bespoke to the care industry and a brilliant opportunity with the benefit of networking and sharing ideas' Registered Manager, Residential Home.

Conclusion 'Highly innovative in its approach which is helping to transform the people we serve by fully engaging front line colleagues' Director of Adult Social Care. We now need to consider sustainability. With funding streams limited there is a commitment from the Local Authority and Clinical Commissioning Group to continue to find ways of ensuring this programme continues beyond December 2020 to ensure the standards of care provision continue to rise with good evidence provided through the five key lines of enquiry documentation.

\section{0-7 PREPARING THE WAY: EVALUATING SUPPORT FOR CHILDREN BEFORE THE DEATH OF A PARENT OR SIBLING}

Alison Penny. Childhood Bereavement Network, London, UK

\subsection{6/bmjspcare-2019-HUKNC.7}

This presentation will introduce the Serious Illness in the Family Service Questionnaires: a new suite of tools for evaluating support for children and young people who are facing the death of someone important in their lives.

Around 24,000 parents die each year, leaving children under 18 (Childhood Bereavement Network, 2016), with 60$75 \%$ of these deaths being expected (End of Life Care Intelligence Network, 2011). Over 10,000 babies, children and young people under 25 die each year, many leaving siblings (Child Bereavement UK, 2017).

The death of a close family member is associated with a range of poor outcomes for children and young people both in the short and medium term and into adulthood, including early mortality, mental and physical health problems and disrupted education (Penny \& Stubbs, 2015). For children bereaved of a parent through cancer, the time before the death is particularly stressful (Siegel et al., 1992). Over the last three decades, a range of services have developed across the UK to respond to bereaved children's experiences and needs (Rolls \& Payne 2003; Rolls \& Payne 2004; Penny, 2011). An increasing number of services - including many hospices - are also offering support to children before a death, where this is possible.

There are considerable challenges to evaluating this type of support. This presentation will share findings from a project that:

- reviewed the areas of concern to families and practitioners about children's needs before a death in the family;

- scoped interventions and built consensus on the aims of support services in the UK;

- reviewed current quantitative measures being used in evaluations in research and practice;

- identified key challenges to evaluating these interventions;

- developed a suite of self- and parent-reported measures to capture changes towards outcomes across hospices and other services working with children facing the death of someone important.
$0-8$

THE DONOR EXPERIENCE: VALUE CREATION AND THE ROLE OF THE DONOR IN SUPPORTER-LED FUNDRAISING

${ }^{1}$ Katie Mitchell, ${ }^{2}$ Anna Ackfeldt. ${ }^{1} J o h n$ Taylor Hospice, Birmingham, UK; ${ }^{2}$ Aston University, Birmingham, UK

\subsection{6/bmjspcare-2019-HUKNC.8}

Background In 2015, a series of scandals exposed aggressive fundraising practices by charities that had caused distress to donors. In contrast, business has understood that the customer experience is vital to their success (Bitner \& Brown, 2008; Lusch, Vargo \& O’Brien, 2007; Martin, 1999). Services marketing theory describes the role of the customer in 'co-creating' their experience to increase customer satisfaction (Grönroos, 1994). Fundraisers now recognise that how donors feel about their experience of fundraising is crucial. Community fundraising is driven by donors who organise and deliver their own fundraising events and experiences. But there is a gap in our knowledge about the role donors play in creating their own experience and the effect on donor satisfaction.

Aim To examine whether academic models derived from services marketing literature can be applied to community and supporter-led fundraising to improve the donor experience. In particular, we examine the concept of value and the role of the donor in value creation.

Methods A literature review was conducted which highlighted the limited research available. Consequently, an exploratory approach was adopted. Empirical primary data was captured through a series of interviews at case study organisations. Three case studies were identified within a specific typology of organisation: hospice charities that fundraise using similar techniques within their local communities.

Results The concept of value-in-use described by Grönroos (1994) and the role of the donor as an independent creator of value in the customer sphere (Grönroos \& Voima, 2013) was supported by the research and can be applied to the donor experience.

Conclusion It has never been more important for fundraisers to deliver an excellent donor experience. Fundraisers must employ coaching and facilitation skills to empower supporters to create value. Further research is required to ascertain how managers of fundraising can deliver service encounters and tangible resources that best facilitate the creation of value for the donor.

\section{Parallel Session 3: Symptom Management and Treatment}

\section{0-9 THE DEVELOPMENT OF A CENTRALISED METASTATIC SPINAL CORD COMPRESSION COORDINATOR SERVICE}

Lena Richards, Vivek Misra, Claire Shanahan. The Christie NHS Foundation Trust, Manchester, UK

\subsection{6/bmjspcare-2019-HUKNC.9}

Background MSCC is estimated to occur in 5-10\% of cancer patients. Prompt diagnosis and treatment is essential to prevent paralysis. Failure to recognise early signs can have devastating implications, including the financial burden of care for patients who become bedbound and with earlier than expected death. 
Aims(s) The service was launched in 2013 covering a population of 3.5 million. It has significantly improved the care of patients who develop this distressing condition across our region. Education is integral to the role and has raised awareness amongst patients and clinicians of the early signs.

Methods The service provides a single point of contact for all health-care sectors. It offers advice and coordinated care based on evidence-based guidelines and a robust network pathway. Patients are triaged and referred for surgery, radiotherapy, systemic treatment or best supportive care. All patients are offered rehabilitation. Data are collected prospectively for service evaluation.

Results Evaluation has demonstrated significant improvements. In the first four years, 2635 patients were referred. 96\% had an MR scan within 24 hours, out of these, 1133 had confirmed MSCC and 345 had impending cord compression. $16 \%$ of patients were treated with surgery (this is a three-fold increase since before the service) and 64\% with radiotherapy, also within 24 hours.

Conclusion Awareness amongst patients and clinicians has significantly increased resulting in timely diagnosis, treatment and rehabilitation. Earlier diagnosis also has the potential to prevent MSCC in the future. Back pain is managed earlier and patients remain ambulant with improved survival, reducing hospital admissions with overall financial benefits.

\section{0-10 METASTATIC SPINAL CORD COMPRESSION PATIENT SURVIVAL FOLLOWING ESTABLISHMENT OF A COORDINATOR SERVICE}

Lena Richards, Vivek Misra, Claire Shanahan. The Christie NHS Foundation Trust, Manchester, UK

\subsection{6/bmjspcare-2019-HUKNC.10}

Background Clinical trials have shown surgery to be superior to radiotherapy for patients with metastatic spinal cord compression (MSCC). We present our results following the establishment of a centralised MSCC service, the aim of which is to coordinate the pathway of patients with MSCC within 15 hospitals that serve a population of 3.5 million people.

Aims The primary aim of this study was to determine the survival time following treatment for metastatic spinal cord compression. The secondary aim was to compare our results against survival times quoted in the medical literature.

Methods Data was prospectively collected and analysed for all patients referred with confirmed MSCC in 2014 and 2015. Patients were triaged by the oncologist and spinal surgeon, based on prognosis and performance status and categorised into four subtypes: (1) best supportive care (2) systematic anti-cancer therapy (3) spinal surgery and post-operative radiotherapy (4) radiotherapy alone.

Results 1253 patients were referred to the MSCC service, of which 540 (43\%) had confirmed MSCC. 73 patients (14\%) received best supportive care (median survival of 32 days), 24 patients (4\%) had systemic anti-cancer therapy (median survival of 637 days), 89 patients (16\%) had surgery (median survival of 377 days) and 354 patients (66\%) had radiotherapy (median survival of 62 days).
Conclusion Our results of 377 days compare favourably to studies by Patchell and Fehling, which found median survivals of 126 days and 230 days respectively following surgery. Our data strongly supports the use of decompressive spinal surgery with instrumented stabilisation for MSCC in carefully selected patients.

\section{0-11 DEACTIVATION OF IMPLANTABLE CARDIAC DEFIBRILLATORS AT END-OF-LIFE: INTEGRATED WORKING IS ESSENTIAL}

${ }^{1}$ Fiona Hodson, ${ }^{2}$ Julia DeCourcey, ${ }^{2}$ Stefan Karwatoski, ${ }^{2}$ Mohammed Albarjas, 'Joy Ross, ${ }^{1}$ Rob George. 'St Christopher's Hospice, London, UK; ${ }^{2}$ King's College Hospital Foundation Trust, London, UK

\subsection{6/bmjspcare-2019-HUKNC.11}

Background The benefits of integrated cardiac and palliative care support for patients with end-stage heart failure (ESHF) is well recognised (Jaarsma, Beattie, Ryder et al., 2009; Hospice UK, 2017). NICE guideline support use of implantable cardiac defibrillators as part of optimal treatment. Guidelines recommend early discussion with 'careful, explicit, shared-decision making' to promote timely deactivation at end-of-life (NICE, 2014; British Heart Foundation, 2007). In practice this presents challenges for both professionals and patient/family, with varying and limited access to deactivation in the community setting.

Methods A case series is presented from a service evaluation of an integrated model of care for ESHF patients. 10/89 patients had implantable cardiac resynchronization therapy defibrillators (CRT-D) and $1 / 89$ an implantable defibrillator (ICD). We present some of the challenges in management of these devices in our palliative cohort.

Results 8/10 devices were deactivated due to acute deterioration of patients; $1 / 10$ patients reported shocks given. Timing of deactivation was arranged according to the patient's clinical condition and wishes, predominately within a month of death. Deactivation occurred in a variety of settings: hospital inpatient (3), hospital outpatient (3), home (1), hospice (1), including visits by electrophysiologists from tertiary centres and temporary use of magnets by clinicians.

Discussions around deactivation of the CRT-D were complex due to previous clinical history and ingrained patient/family perceptions. Discussions were always multiprofessional; one case was taken to the Hospice Clinical Ethics Committee. One patient had the defibrillator function deactivated and was subsequently resuscitated by the Ambulance Service when he went into fast atrial fibrillation.

Family involvement and agreement with decision making was key, as was joint discussions by different professionals across care settings including those who had known the patient longest.

Conclusion These cases illustrate the need for further discussion and protocols regarding the time and place of deactivation of defibrillators at end of life, and also the challenges for professionals of having resuscitation discussions with these patients and importantly their families. 